\title{
Genetic Algorithms for Reflective Filters Design
}

\author{
E. Herbert Li* and A. B. Djuri $\{$ i $\}$ \\ Dept. of EEE, University of Hong Kong, Pokfulam Road, Hong Kong
}

\begin{abstract}
A genetic algorithm (GA) with adaptive mutations has been employed for the design of Bragg reflectors. The algorithm enables three different design types a) composition and thickness of each layer are optimized b) two compositions are chosen for the two alternating materials, and thickness of each layer is optimized c) composition and thickness of two layers are chosen and the pair is repeated. The algorithm enables finding the optimal design for two chosen incident and final media, and it is capable of taking into account the existence of a finite, optically thick substrate. The algorithm is very versatile and can be applied for the design of refractive filters using various materials. We have demonstrated application of this algorithm to the design of $\mathrm{Al}_{\mathrm{x}} \mathrm{Ga}_{1-\mathrm{x}} \mathrm{N}$ reflectors, as well as organic and dielectric reflectors.
\end{abstract}

Index terms - genetic algorithms, Bragg reflectors

\section{INTRODUCTION}

Nitride materials have attracted lots of attention recently for their potential applications in optoelectronic devices. Design of highly reflective $\mathrm{Al}_{\mathrm{x}} \mathrm{Ga}_{1-\mathrm{x}} \mathrm{N}$ reflectors is crucial for nitride based vertical cavity surface emitting lasers. AlN/GaN reflectors would theoretically give the highest reflectance, but in practice this is not the case due to scattering losses $[1,2]$. The design algorithm used here allows imposing constraints on the composition difference of the neighbouring layers, either with a penalty function or with narrowing the boundaries for possible compositions. This feature is important because the large lattice mismatch between GaN and AlN can cause poor surface morphology, so measured reflectivity would be lower than the calculated one due to the surface roughness. Two material systems have been investigated: air/sapphire/ $\mathrm{Al}_{\mathrm{x}} \mathrm{Ga}_{1-\mathrm{x}} \mathrm{N}$ reflector/ and $\mathrm{GaN} / \mathrm{Al}_{\mathrm{x}} \mathrm{Ga}_{1-\mathrm{x}} \mathrm{N} / \mathrm{air}$. It has been found that the all three design types exhibit either better or similar characteristics compared to the conventional sequence of alternating quarter-wave layers with low and high refractive index. Better characteristics of GA optimized design are particularly pronounced in the case were a substrate is present.

Organic materials have recently attracted attention for potential applications in organic light emitting diodes (OLEDs). It has been shown that the performance of an

\footnotetext{
*E_mail: ehli@eee.hku.hk
}

OLED can be improved if a diode is placed between two reflectors. Usually, one reflector is the top metal electrode, while the other one is either another metal layer or the dielectric stack, with hole-injecting contact placed inside the cavity in the latter case [3]. An all-organic microcavity light emitting diode based on conjugated polymers where the Bragg reflector has been realized with semiconducting polymers has been demonstrated [4]. In this device injecting anode is placed outside the cavity, thus improving its quality factor. To our knowledge, no such device has been made using small molecules. We demonstrate here the calculations of the reflectance characteristics of the reflectors made of organic materials. Two designs have been investigated: one for blue-green spectral region (suitable for $\mathrm{Alq}_{3}$ based devices) and one in red spectral region (suitable for europium complex based devices). Results for the design of dielectric based reflective filters in the same spectral regions are also presented. Most of the materials used in the design of organic reflective filters have been demonstrated to serve as hole-transport or holeinjection layers, so such reflectors have potential use in microcavity-based OLEDs. However, the true influence of such all-organic reflector to the carrier transport in OLEDs needs further investigation. It has been shown that the genetic algorithm based reflective filter design method is highly versatile and suitable for the design of reflective filters using a variety of materials.

\section{DESCRIPTION OF THE ALGORITHM}

In order to implement a GA, we must define the coding scheme, initial population generation, and selection, crossover and mutation operators. In this work we have used elite genetic algorithm with adaptive mutations (EGAAM) [5]. Floating-point coding scheme [5-7] has been employed. Each layer is characterized with two real numbers, alloy composition and layer thickness. In this coding scheme, each gene has the value of the corresponding variable $p(k), k=1, n_{v}$, where $n_{v}$ is the number of variables. The number of variables differs for three design procedures. In the design procedure a), composition and thickness of each layer are chosen independently, and the total number of variables is equal $2 \times n_{l}$, where $n_{l}$ is the number of layers. In design procedure $b$ ), two materials are used while layer thickness of each layer is still determined independently, so that the number of variables is $n_{l}+2$, for two layer compositions and $n_{l}$ layer thickness parameters. For design procedure c), the algorithm chooses only alloy 
composition and layer thickness for two layers, which are repeated required number of times, so that the number of variables is 4 regardless of the number of layers. This design procedure is the closest to the conventional quarterwave design. The advantage of this procedure over the conventional design is that the constraints can be introduced, and optimal design found within given constraints. The values of variables in the initial population are in all cases generated according to the equation [5]

$$
p(k)=p_{l}(k)+\left(p_{u}(k)-p_{l}(k)\right) \times r,
$$

where $r$ is a random number $r \in[0,1]$, and $p_{l}(k)$ and $p_{u}(k)$ are initially set lower and upper boundary, respectively. In such a manner, confinement of the variables in the specified domain is achieved in order to insure that variables have physical meaning, i.e. that all the thickness values are positive and all the compositions $0 \leq x \leq 1$.

EGAAM employs elitist selection mechanism [810]. In this selection mechanism, $P_{s}$ percent of the strings in the current population enter the next population unchanged, while $P_{c}$ percent of the strings in the new population are generated by crossover between the parent strings. $P_{s}$ and $P_{c}$ are initially specified values. Parent strings on which crossover is performed are chosen fitness proportionally. The probability of a string having offspring in the next generation is inversely proportional to [5]

$$
F(i)=\frac{f(i)}{\sum_{i} f(i)},
$$

where $f(i)$ is the fitness value of $i$-th string.

Operation of crossover exchanges subsets of elements between two parent strings. If the subsets consist of adjacent elements, it is an "ordered combination" crossover, while in "uniform combination" crossover each element is randomly chosen [10]. EGAAM employs "uniform combination" crossover, with a number of elements to be exchanged randomly generated at each crossover operation [5]. After the number of elements to be exchanged has been generated (which is always greater than the specified minimal number of elements), random integers determining positions of elements to be exchanged are generated and elements on those positions are swapped.

Mutation ensures that the population diversity is maintained, thus preventing quick convergence to a local minimum. If the mutation probability is too large, mutation no longer improves the performance of the algorithm, since it enables losses of genetic information which can cause poor convergence [12]. EGAAM employs adaptive mutation concept [5] in order to overcome the phenomenon that real-coded GA can be blocked from further progress $[13,14]$. In adaptive mutation scheme, $P_{m}$ percent of completely new strings are introduced in every generation. The new strings are generated in the same way as during initial population generation, but within narrowed boundaries to provide that more strings are generated in the area where a solution is expected. The boundaries are narrowed according to equations [5]

$$
\begin{aligned}
& p_{\text {new-u }}(k)=p_{\text {old }-u}(k)-c\left(p_{\text {old }-u}(k)-\mu(k)\right) \\
& p_{\text {new-l }}(k)=p_{\text {old }-l}(k)+c\left(\mu(k)-p_{\text {old }-l}(k)\right),
\end{aligned}
$$

where $\mu(k)$ is the average value of parameter $p(k)$ in the current population, and $c$ is a predetermined positive number $c<1$. To prevent excessive narrowing of the boundaries, lower boundary was limited to be less than maximal allowed lower boundary, and upper boundary was limited to be greater than minimal allowed upper boundary.

The objective function represents a measure of the quality of the obtained solution. To achieve the desired reflectance dependence on the wavelength $\lambda$, we have used the following objective function

$$
F=\left\{\sum_{i=1}^{n_{p}}\left(R_{c}-R_{d}\right)^{2} \exp \left(-\frac{\left(\lambda-\lambda_{0}\right)^{2}}{2 \sigma^{2}}\right)\right\}+\Delta,
$$

where $n_{p}$ is the number of points in which we calculate the reflectance $R_{c}, R_{d}$ is the desired reflectance, and $\lambda_{\theta}$ is the wavelength where peak reflectivity should be achieved. $\Delta$ is the penalty function, whose value is set to zero if the composition difference constraints are satisfied, or set to a specified value $10 \leq \Delta \leq 100$ if the composition difference between adjacent layers is larger than the required value. The desired reflectance is equal to 1.0 in the range $\left[\lambda_{0}-\Gamma, \lambda_{0}+\Gamma\right]$ where $\Gamma$ is the half-width of reflectance peak, and equal to zero outside that range. The exponential factor in Eq. (6) provides enhancement of the peak at $\lambda_{\theta}$.

\section{RESULTS AND DISCUSSION}

Reflectance in all cases was calculated using matrix method, and since substrates are transparent, the reflectance value was corrected for the incoherent substrate back side reflections. The index of refraction of $\mathrm{Al}_{\mathrm{x}} \mathrm{Ga}_{1-\mathrm{x}} \mathrm{N}$ as a function of wavelength (energy $\hbar \omega(\mathrm{eV})=1239.85 / \lambda(\mathrm{nm}))$ and composition can be described with a simple expression in the spectral region of interest [15]:

$$
\begin{gathered}
n^{2}(\omega)=C(x)+A(x) y^{-2}(2-\sqrt{1+y}-\sqrt{1-y}), \\
y=\frac{\hbar \omega}{E_{g}}, \quad A(x)=3.17 \sqrt{x}+9.98 \\
C(x)=-2.2 x+2.66 \\
E_{g}(x)=E_{g}(\mathrm{GaN})(1-x)+E_{g}(\mathrm{AlN}) x-b x(1-x)
\end{gathered}
$$

where $E_{g}(\mathrm{GaN})=3.42 \mathrm{eV}, E_{g}(\mathrm{AlN})=6.13 \mathrm{eV}$ and $b=1.3 \mathrm{eV}$. However, in order to take into account the absorption in the spectral region of interest for alloys $0 \leq x \leq 0.4$ it is convenient to rewrite the Eq. (6), with $N^{2}(\omega)=\varepsilon(\omega)=\varepsilon_{1}(\omega)+i \varepsilon_{2}(\omega)$, as [16]: 


$$
\begin{gathered}
\varepsilon_{1}(\omega)=C(x)+A(x) y^{-2}(2-\sqrt{1+y}-\sqrt{1-y} H(1-y)),(10) \\
\varepsilon_{2}(\omega)=A(x) y^{-2} \sqrt{y-1} H(y-1),
\end{gathered}
$$

where $H(x)=1, x \geq 0$ and $H(x)=0, x<0$. The real and imaginary part of the index of refraction are then readily calculated. The optical constants of sapphire in the spectral region of interest can be described with $k_{s}=0.0$ and $n_{s}=1.752+5497.26 / \lambda^{2}$, which describe well the tabulated data [17]. If no constraints are imposed, the algorithm will produce an alternating sequence of GaN/AlN layers for all three design methods a), b), and c). This is because $\mathrm{GaN}$ and AlN have the largest possible difference in refractive indices, and therefore the calculated reflectivity of a GaN/AlN reflector would be the highest for a given number of layers. However, due to practical limitations in the growth of GaN/AIN reflectors, we will limit our discussion to the cases where the composition difference between adjacent layers is $\leq 0.5$. We compare the calculated reflectance of conventionally designed reflectors consisting of alternating layers of $\mathrm{Al}_{\mathrm{x}} \mathrm{Ga}_{1-\mathrm{x}} \mathrm{N} / \mathrm{Al}_{\mathrm{y}} \mathrm{Ga}_{1-\mathrm{y}} \mathrm{N}$ with layer thickness determined with $d_{i}=\lambda_{0} / 4 n_{i}$, where $n_{i}$ is the refractive index of the layer

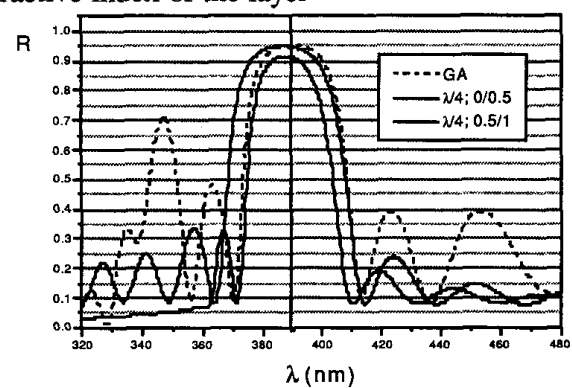

Fig. 1 The reflectance of a 30 layer reflector for the structure air/sapphire/reflector/GaN. Dashed line denotes GA optimized design with composition difference $\leq 0.5$, while thick and thin solid lines denote $\mathrm{Al}_{0.5} \mathrm{Ga}_{0.5} \mathrm{~N} / \mathrm{GaN}$ and $\mathrm{AlN} / \mathrm{Al}_{0.5} \mathrm{Ga}_{0.5} \mathrm{~N}$ quarter-wave reflectors, respectively.

In Fig. 1, dashed line denotes GA optimized design with method c) and composition difference $\leq 0.5$ for structure air/sapphire/reflector/GaN, while thick and thin solid lines denote $\mathrm{Al}_{0.5} \mathrm{Ga}_{0.5} \mathrm{~N} / \mathrm{GaN}$ and $\mathrm{AIN} / \mathrm{Al}_{0.5} \mathrm{Ga}_{0.5} \mathrm{~N}$ quarter-wave reflectors, respectively. Fig. 2 shows the obtained results for the structure $\mathrm{GaN} /$ reflector/air. In both cases, calculations for 30 layers are presented. Light is incident from GaN. It can be clearly observed that the value of the reflectance peak in the conventional design depends on the composition of the layers and not only on the composition difference between the layers. Therefore, although GA optimized design using method a) produces a sequence of $\lambda / 4$ alternating layers similar to the conventional design, the important advantage of this method is that it chooses automatically which compositions should give the highest reflectance for given composition difference.

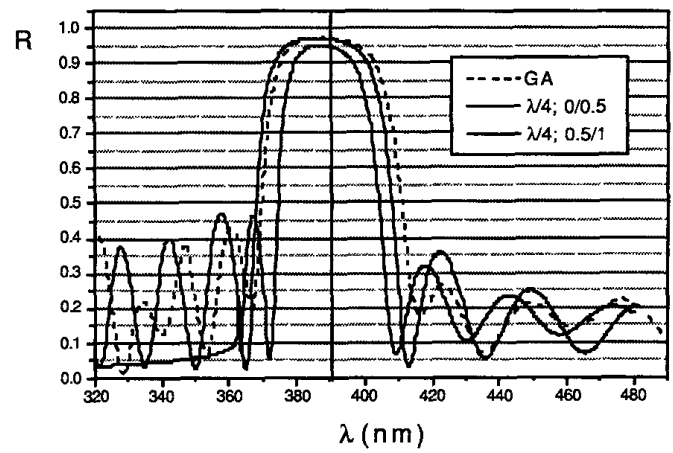

Fig. 2 The reflectance of a 30 layer reflector for the structure GaN/reflector/air. Dashed line denotes GA optimized design with composition difference $\leq 0.5$, while thick and thin solid lines denote $\mathrm{Al}_{0.5} \mathrm{Ga}_{0.5} \mathrm{~N} / \mathrm{GaN}$ and $\mathrm{AIN} /$ $\mathrm{Al}_{0.5} \mathrm{Ga}_{0.5} \mathrm{~N}$ quarter-wave reflectors, respectively.

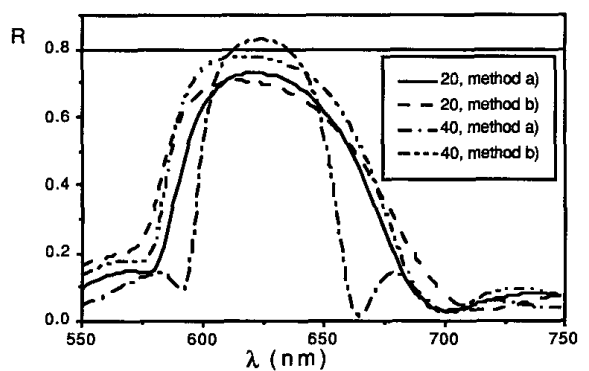

Fig. 3 Reflectance of filters for design type b) and c).

We have designed organic Bragg reflectors for peak wavelengths in the blue-green spectral region and red spectral region. The results are better in the red spectral region because of larger number of available materials which do not absorb in that spectral region. The list of available materials presently includes $N, N^{\prime}$-diphenyl-N,N'bis(3-methyl-phenyl)-1,1'biphenil-4,4'diamine (TPD), 3,4,9,10-perylenetetracarboxylic dianhydride (PTCDA), indanthrenyellow GK (manufactured by Department of Chemistry, University of Technology Dresden), copperphthalocyanine (CuPc), and $\mathrm{N}, \mathrm{N}^{\prime}$-dimethylperylene-3,4: 9,10-dicarboximide. The index of refraction data for 3,4,9,10-perylenetetracarboxylic dianhydride, indanthrenyellow GK (manufactured by Department of Chemistry, University of Technology Dresden), copper phthalocyianine, and $\mathrm{N}, \mathrm{N}^{\prime}$-dimethylperylene-3,4:9,10dicarboximide have been modeled using a modified oscillator model $[18,19]$. The index of refraction of ITO was calculated using the model given in [20]. The experimental data for TPD [21], have been modeled with the refractive index dependency in the form $n=A+B / \lambda^{2}$, and the same expression was used to model refractive index of glass. Due to small differences in the refractive index of the organic materials considered, method c) does not produce good results except for very large number of 
layers. Therefore, we will show here the results of asymmetric designs, procedures a) and b). Figure 3 shows the reflectivity of GA optimized filters using methods a) and b) for 20 and 40 layers. It can be observed that the method a) obtains higher value of the reflectance peak compared to method b). This is to be expected due to greater flexibility of the design method a). Figure 4 shows the reflectance of a filter design using method b) for dielectric materials (solid line) and organic materials (dashed line). The index of refraction of the considered dielectric materials $\left(\mathrm{SiO}, \mathrm{SiO}_{2}\right.$, and $\left.\mathrm{Si}_{3} \mathrm{~N}_{4}\right)$ has been modeled using a model described in [22]. It can be observed that the height of the reflectance peak is larger for the reflector made of dielectric materials, as well as the half-width of the peak. This is due to larger difference in the refractive index of the available materials. Figure 5 shows the reflectivity of GA optimized filters using methods a) and b) for 30 and 40 layers, designed for the peak wavelength in the blue-green spectral region.

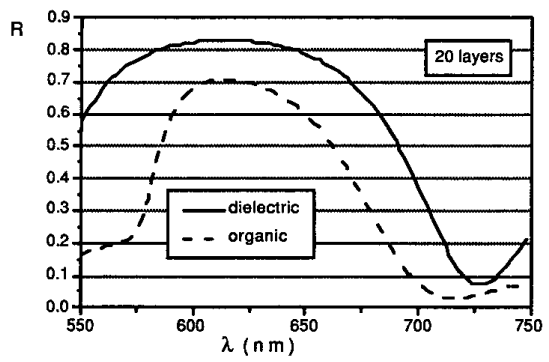

Fig. 4 Reflectance of filters designed with method b) for 20 layers for dielectric and for organic materials.

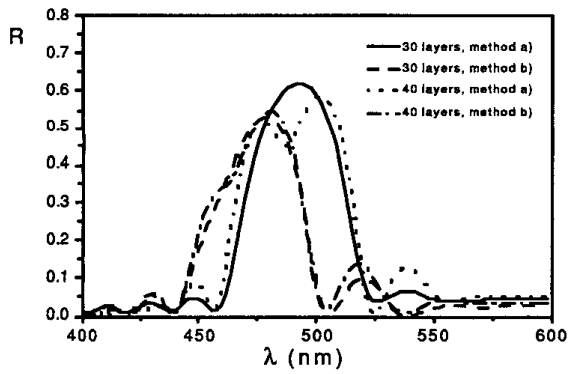

Fig. 5 Reflectance of filters for design type a) and b).

\section{CONCLUSION}

We have presented a genetic algorithm based method for the design of the reflective filters. The described design method represents a useful tool in filters design since it is highly versatile, i.e. it enables easy changes of design objective by simple manipulations of the objective function, and the materials involved can be easily changed. The method has been applied to $\mathrm{Al}_{\mathrm{x}} \mathrm{Ga}_{1-\mathrm{x}} \mathrm{N}$ Bragg reflectors, organic and dielectric Bragg reflectors.

\section{ACKNOWLEDGEMENTS}

The authors would like to thank the RGC earmarked grant of Hong Kong and the University of Hong Kong CRCG research grant for financial support.

\section{REFERENCES}

[1] T. Shirasawa et al, J. Crystal Growth, vol. 189/190, pp.124-127, 1998.

[2] H. M. Ng et al., Appl. Phys. Lett., vol. 74, pp. 1036$1038,1999$.

[3] K. Neyts et al., J. Opt. Soc. Am. B, vol. 17, pp. 114$119,2000$.

[4] P. K. H. Ho et al., Science, vol. 285, No. 5425, pp. 233-236, 1999.

[5] A. B. Djuri\{i\}, Opt. Commun., vol. 151, pp.147159,1998 .

[6] K. P. Wong and Y. W. Wong, Proc. ANZIIS-93, Perth, Western Australia, 1993, pp. 512-516.

[7] K. P. Wong and Y. W. Wong, IEE Proc. Gen. Transm. Distrib., vol. 141, pp. 507-513, 1994.

[8] R. Vemuri and R. Vemuri, Elec. Lett., vol. 30, pp. 1270-1272, 1994.

[9] S. H. Clearwater and T. Hogg, Artificial intelligence, vol. 81, pp. 327-347, 1996.

[10] R. R. Brook et al., Artificial intelligence, vol. 84, pp. 339-354, 1996.

[11] F. Curatelli, Int. J. Electronics, vol. 78, pp. 435-447, 1995.

[12] D. Raynolds and J. Gomatam, Artificial intelligence, vol. 82, pp. 303-330, 1996.

[13] D. E. Goldberg, Complex Systems, vol. 5, pp. 139$167,1991$.

[14] K. Deb and R. B. Agrawal, Complex Systems, vol. 9, pp. 115-148, 1995.

[15] D. Brunner et al., J. Appl. Phys., vol 82, pp. 50905096, 1997.

[16] S. Adachi, Phys. Rev. B, vol. 41, pp. 3504-3508, 1990.

[17] F. Gervais, in Handbook of Optical Constants of Solids II, E. D. Palik (Ed.), Academic, San Diego, CA, 1991, pp. 761-775.

[18] A. B. Djuri\{ i\} et al., J. Opt. A: Pure Appl. Opt. vol. 2, pp. 458-464, 2000.

[19] A. B. Djuri $\{$ i\} et al., Opt. Commun., vol 183, pp. $123-132,2000$.

[20] S. Laux et al., Thin Solid Films vol. 335, pp. 1-5, 1998.

[21] F. G. Celii et al., J. Electron. Mater. vol. 26, pp. 366371, 1997.

[22] A. B. Djuri\{i\} and E. H. Li, Appl. Opt. vol. 37, pp. 5291-5297, 1998. 